\title{
A HYBRID AHP/FST MODEL FOR REGIONAL AIRCRAFT EVALUATION
}

\author{
Giuseppe Bruno \\ Dept. of Industrial Engineering (DII) \\ University of Naples Federico II \\ Naples, Italy \\ E-mail: Giuseppe.bruno@unina.it \\ Emilio Esposito* \\ Dept. of Industrial Engineering (DII) \\ University of Naples Federico II \\ Naples, Italy \\ E-mail: emilio.esposito@unina.it
}

\author{
Andrea Genovese \\ Logistics and Supply Chain Management Research Centre \\ University of Sheffield \\ Sheffield, UK \\ E-mail: a.genovese@ shef.ac.uk
}

\begin{abstract}
This paper presents a hybrid model for regional aircraft evaluation, based on the two main approaches proposed in the literature, the Analytic Hierarchy Process (AHP) and the Fuzzy Set Theory (FST). The proposed model combines the strengths of the two approaches, overcoming some of their weaknesses. AHP is preserved for criteria weights determination whereas FST is adopted to deal with aircraft performances. The hybrid model is implemented to compare three regional aircrafts. The results highlight how the proposed model can be useful both for manufacturing companies, in their process of designing future regional aircraft, and for airlines to support the choice of the aircraft that better satisfy their requirements.
\end{abstract}

Keywords: AHP, FST, regional aircraft, evaluation

\section{Introduction}

In the contemporary global market characterized by an increasing attention to the environmental aspects (OPEC, 2008; WRI, 2008), airlines and manufacturing companies can no longer consider the air transport only as a cost-oriented problem. In addition to traditional aspects, such as operative costs and technical performance, others, such as environmental impact, green performance, and comfort are becoming more and more crucial (Braathen, 2005; ETS, 2005; EU, 2007, 2008; ICAO, 2003; Hope, 2005; Newson \& Cairns, 2006).

For airlines the choice about the aircraft to purchase, is not a simple problem of efficiency, mainly oriented to minimize operative costs, but it involves either efficiency or effectiveness, i.e. the choice of the aircraft that assures the best combination among costs, technical characteristics, comfort, and environmental impact (Canaday, 2005; Doganis, 2006; Stonecipher, 2008).

On the other side, large manufacturer companies, before the launch of a new programme needs huge investments, have to clearly identify the set of characteristics that better satisfy the airlines requirements. The risk is the failure of the new programme, the lost of relevant investments and the consequent decline of the company brand (D. Bernstein, 2008; Ferreri, 2003; Wall, 2008).

In this context the aim of this paper is to propose a model for regional aircraft (GRA) evaluation, based on the investigation of the airlines needs, that considers not only traditional characteristics (direct and indirect operative costs and technical performance such as the cruise speed) but also includes a variety of aspects whose importance is strikingly increasing, such as green norms and comfort. The proposed model can be useful both for airlines, in their process of selecting the most suitable aircraft for their fleet, and for manufacturing companies in their process of designing future regional aircrafts.

These considerations underline that in the air transport industry the cost-benefit analysis is becoming a multi-attribute problem, typified by a high complexity level in which a variety of quantitative and qualitative factors play a crucial role. Moreover, although the literature is rich of contributes suggesting tools to deal with this problem, only recently airlines and manufacturing company are focusing their attention on this problem.

In this context, the paper starts from the evaluation of the two main approaches proposed in literature to deal with the evaluation problem, the Analytic Hierarchic Process (AHP) and the Fuzzy Set Theory

\footnotetext{
* Corresponding author
} 
(FST), proposing a hybrid model which overtakes some of their weaknesses and combines some of their strengths. In particular the AHP approach is preserved for criteria weights determination because it ensures to keep track of the differences between different criteria thanks to pair-wise evaluations between them; the FST approach, instead, is adopted to deal with aircraft performances because it allows to represent the vagueness associated to criteria and to indicate the "nuances" of airlines perceptions about aircraft performances without losing information (Saaty, 1980, 2001; Zadeh, 1965).

The usability of the hybrid model for the players of the air transport industry and its adaptability to environments characterized by complexity, high tech level, and increasing requirement of sustainability, is investigated through an empirical study focused on aircrafts for regional transport. The results provided by the application of the model application triggered some interesting debates regarding the model itself and the general purpose which has to drive hybrid model implementation to guarantee successful results.

The paper is organized in the following sections. In the next section the hybrid model is introduced and described. In the successive section the proposed model is implemented and a case study related to the evaluation of three regional aircrafts are analyzed and discussed. Finally, some conclusions are drawn.

\section{A Hybrid Model for regional aircraft evaluation}

From the assessment of AHP and FST approaches some weakness and strengths emerge. In particular there are some distortions introduced by AHP and FST techniques in the perceptions, evaluation and computation respectively of performances and weights associated to criteria adopted in the supplier selection process.

AHP model biases the performances associated to the criteria for the broad sensitivity of the results given by model implementation from the qualitative ranges defined by decision makers for the evaluation criteria. Moreover, since these ranges are, generally, treated as flat scales where all the values belonging to the same range assume exactly the same relevance, there is no way to keep track of distances between values measured. In particular, the distances between values belonging to the same range, but positioned at its extremes, are ignored; hence a strong variation is nullified. Similarly it is not possible to mark if some values are close among them but around the border of adjacent qualitative ranges; in this case little differences between values are spread, becoming much bigger than how they really are. For these reasons, when AHP is adopted for supplier performance evaluation, differences are not properly tracked and the ending outcomes of the model may appear significantly altered. Indeed, from a general point of view, Triantaphyllou and Mann (1995) suggest that the recommendations made by the AHP should not be taken literally. In matter of fact, the closer the final priority values are with each other, the more careful the user should be. Furthermore, in the extant literature, several scholars have expressed some concerns about practical applications of AHP-based methods for modelling and solving real world problems. Harkar and Vargas (1990) and Perez et al. (2006) show that AHP suffers from the so-called "rank reversal" problem. Indeed, because priorities associated with alternatives depend on what alternatives are considered, hence, even adding or deleting irrelevant alternatives can lead to change in the final rank. When FST approach, instead of AHP, is applied for dealing with criteria performances, the distortions illustrated above are almost eliminated. Indeed, in FST models, the qualitative scale defined for each criterion is not treated as a flat scale, as membership functions are defined for each one of the levels. In this way, a fuzzy variable is associated with each crisp numerical value of the indicators associated to the evaluation criteria, This fuzzy variable keeps track of the degree of membership of the measured value to each defined qualitative range; hence, biases introduced by AHP approach are almost overtaken. On the other side, from an accurate investigation on FST model, and in particular on its application in real-world practice, some other findings come up. When decision-makers are inquired to state judgments about the weights associated to different criteria, a kind of a flattening/overestimating effect of weights assessment is triggered. Firstly differences between levels of importance of criteria are lost and then lots of criteria are overestimated. This happens for the uncontrolled decision-makers propensity to judge criteria importance equally high or very high for all the criteria considered when absolute qualitative judgments are inquired. When AHP is applied for weights determination, the drawbacks illustrated above are overcome thanks to pair-wise evaluations between criteria (Saaty, 1980) that allow detecting even little differences perceived by decision makers about importance assigned to different criteria.

Therefore, AHP model appears relevantly suitable for weights determination, meanwhile for performance evaluation it leads to some biased results; FST model, instead, seems to fit significantly for performance estimations, but on the other side, for weights assessment it introduces some distortions as well. For this reason, we propose a hybrid model for the regional aircraft evaluation, which combines the methodology to determine criteria weights typical of the AHP approach with performances drawn from a FST based model. The actual usability and adaptability of the model in practical applications is investigated through an empirical study described in the next section. In particular, the implementation of the model is characterized by the following steps.

Step 1. As indicated in fig. 1 the starting point of hybrid approach is represented (as in the case of the AHP and FST-based approaches) by the identification of airlines needs.

Step 2. After the identification of airlines needs, the characteristics of the formal model are then defined in terms of evaluation criteria, sub-criteria, alternatives and decision makers. 
Step 3. Once criteria and sub-criteria have been identified, alternatives and decision makers are arranged according to a hierarchical scheme, according to the AHP approach.

Step 4. The determination of the weights is performed following an AHP approach; in particular, after the definition of the hierarchical scheme, relative importance of the evaluation criteria are evaluated through the pair-wise comparison method according to Saaty (1980) scale.

Step 5. Consistency of the obtained pair-wise matrices is then verified.

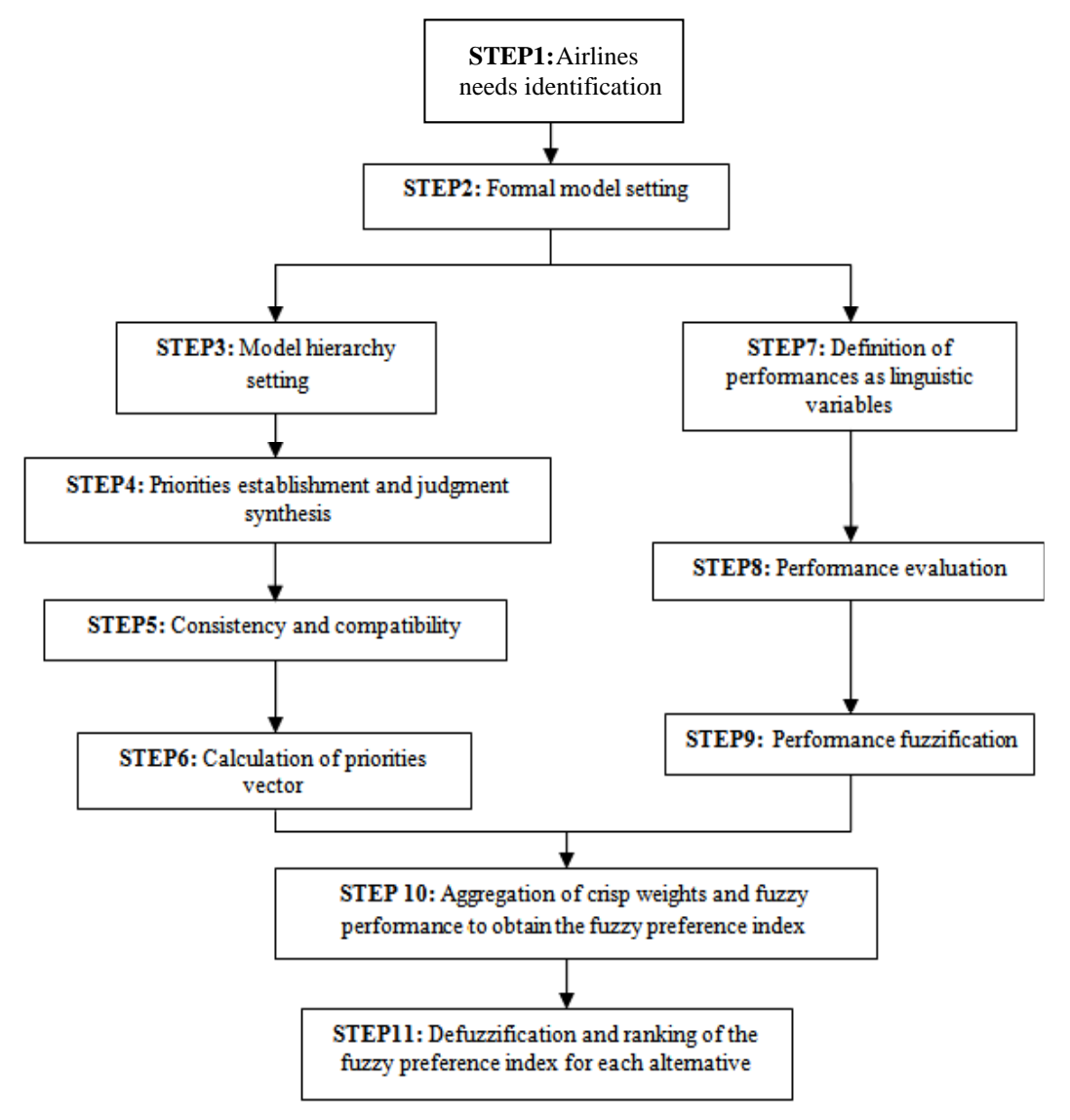

Figure 1. Hybrid, step by step approach

Step 6. The final matrices are then utilised to derive a coherent vector of priorities corresponding to criteria weights.

Step 7. The performance evaluation an FST based approach is proposed; in particular, criteria performances are defined as linguistic variables on five qualitative levels: very poor (VP), poor (P), medium $(\mathrm{M})$, good $(\mathrm{G})$ and very good (VG).

Thereafter, a procedure for membership function determination has to be utilised. From the analysis of literature emerges that the determination methods can be categorized as being manual or automatic. This classification is reported in the Table 1.

Table 1. Classification of approaches for determining the membership function

\begin{tabular}{cc}
\hline Manual & Automatic \\
\hline Frequencies & Artificial neural networks \\
Direct estimation & Genetic algorithms \\
& Deformable prototypes \\
& Gradient search \\
& Inductive reasoning \\
\hline
\end{tabular}

In particular Watanabe (1979) asserted that there are two main manual methods for determining the membership functions: use of frequencies and direct estimation. The frequency method obtains a membership function by measuring the percentage of members of a group (typically experts in a particular domain) who answers yes or not to a question about whether or not an object belongs to a particular set. Direct estimation methods, instead, are based on asking to grade an event on a scale. Watanabe (1979) conducted some experiments on the two approaches concluding that the use of direct estimation is the preferred route. The automatic generation of membership functions covers, instead, a wide variety of different approaches. In general, what makes automatic generation different from the manual methods is that either the expert is completely removed from the process or the membership functions are 'fine tuned' based on an initial guess by the expert. It is difficult to categorize automatic 
approaches, for this reason the classification is made on the used techniques rather than application or methods. Lately, there is a strong emphasis on the use of modern soft computing techniques, in particular for genetic algorithms and neural network.

Starting from this literature framework, we opted for the manual direct estimation method which appears the most suitable and allows to exploit all the information collected from the empirical study.

In particular membership functions are derived by direct estimation starting from three assumptions:

- knowledge acquisition is derived from group of experts, hence multiple points of views are considered for membership function determination;

- the collected knowledge is formalising by fixing numerical ranges corresponding to the values VP, P, M, G, VG estimated for each evaluation criterion;

- trapezioidal and triangular membership functions are selected to represent the term sets. These membership function shapes were judged by the research group as being the most suitable ones for representing the available information.

Given these assumptions, the membership function of the fuzzy numbers representing the terms VP, P, M, $\mathrm{G}, \mathrm{VG}$ associated to the linguistic variable performance, are supposed to be estimated by direct estimation in the way depicted below.

The range where the trapezoidal membership function assumes maximum value (equal to 1), is defined on the range corresponding to the intersection or nearly intersection of the judgments collected from multiple decision makers; the border values (where the trapezoidal membership function assumes values equal to 0 ), instead, are labeled on the extreme values of the range given by the union of the judgments collected from them.

Step 8. Across the following steps depicted in fig. 1, performance evaluation consists of measurement of indicators associated to criteria, through an appropriate data collection process.

Step 9. Performance fuzzification, instead, stands for translation of numerical values given by indicators in fuzzy numbers. In this case the numerical values measured for each criterion are compared to the term set of the linguistic variables defined for it. The values of output membership functions are combined according with the inferred weights of the members through a fuzzy weighted operator. The result of this procedure is a fuzzy number translating the crisp value measured for that criterion. This procedure is assumed to be applied for all the criteria of hierarchy directly estimated to make the evaluation.

Step 10. As depicted in fig. 1, fuzzy performance and crisp weight need to be combined to end up in the final vendor rating. The issue here is represented by the intent to pick the fuzzy aggregation operator in a way to avoid to spread the entropy related to fuzzy numbers when they are combined. The first operators introduced by Zadeh (1965) are complementation, intersection and union. However, the degree of compensation through which humans aggregate criteria is not expressed by these operators. There are others that more accurately represent human decision making; fuzzy weighted mean operator is one of them. Weighted mean operator is a convex composition of several fuzzy sets with coefficients which indicate the 'percentage' of a given set in the aggregation. It allows to combine fuzzy variables coherently with how human decision making, and, at the same time, it is also an operator simple to handle which does not require time expensive or complex calculations. For these reasons it is judged as the most suitable one to adopt for weights, performances and combined aggregation.

Step 11. The de-fuzzification of trapezoidal fuzzy numbers representing the score associated to the different alternatives represents the last step associated to hybrid model implementation. It appears fundamental to translate the final fuzzy score in a crisp number in order to profile the final rank of the alternatives and to identify the best one. Also in this case literature is investigated to figure out the different methods which can be adopted for de-fuzzify fuzzy numbers; combining literature results with the requirements of the specific context of aircraft evaluation the methods for de-fuzzification is selected. De-fuzzification techniques can be classified in three main categories (Saletic et al., 2002): distribution techniques, maxima techniques, area techniques.

The characteristic of distribution technique is that the output fuzzy set membership function is treated as a distribution, for which the average value is evaluated. For this heuristic approach, the output has continuous and smooth change for input variables changes; hence the continuity for these kinds of techniques is high. The basic technique of this group is the center-of-gravity technique (COG). Due to continuity and, often, smoothness of changes of de-fuzzified values, this technique is used with fuzzy controllers. The best known techniques extended from the COG technique are mean-of-maxima (MeOM); basic de-fuzzification distributions (BADD); generalized level set de-fuzzification (GLSD).

Maxima techniques give as a result of de-fuzzification an element from a fuzzy set core. A fuzzy set core consists of elements of the universe of discourse on which that set is defined with the highest degree of membership to the fuzzy set. As the basic representative of that group, the first-of-maxima technique (FOM) can be considered but there are also other techniques such as middle-of-maxima (MOM), last-ofmaxim (LOM), and random-choice-of maxima (RCOM). These techniques are convenient for the general fuzzy expert systems. They are computationally efficient; as matter of fact they belong to the group of the fastest de-fuzzification techniques, because they require passing through values of the core, only. 
Area de-fuzzification techniques use area under the membership function to determine the defuzzification value. The center-of-area technique (COA) belongs to this group of techniques. This method is fast, because only simple operations are used in it, it gives continual change of defuzzification value; hence it is convenient to be used in fuzzy controllers.

Table 2 gives a summary of the features of these three basic defuzzification classes on the base of continuity, computational complexity and domain of applicability.

Table 2. Summary of features of the three defuzzification categories

\begin{tabular}{l|ccc}
\hline & Distribution techniques & Maxima techniques & $\underline{\text { Area techniques }}$ \\
\hline $\begin{array}{l}\text { Continuity } \\
\text { Computational Complexity }\end{array}$ & Yes & No & Yes \\
Domain of applicability & High & Low & High \\
& Fuzzy controllers & Fuzzy expert systems, & Fuzzy controllers \\
\hline
\end{tabular}

As it emerges from table 2, distribution and area techniques are suggested for use in fuzzy controllers; the maxima techniques are suggested for use in general fuzzy expert systems and fuzzy decision-making systems. For these reasons and also for the low computational complexity which characterizes them, maxima technique and in particular the middle of maxima de-fuzzification method is judged as the most suitable to defuzzify aircraft ratings, hence to profile the final ranking of the alternatives.

\section{General Remarks}

The hybrid model for aircraft evaluation detailed above was characterized with the intent to combine AHP and FST strengths, overcoming some of their weaknesses; anyway it does not claim to be perfectly foolproof but present itself some relevant strengths and residual weaknesses. Hybrid model strengths and weaknesses are identified and summarized in table 3.

As indicated in the tab. 3 the main issues of hybrid approach are related to the time spending procedure to carry out the investigations and collect all the information required. Dependence of the final results from some decision dealing with fuzzy holds over. This means that the definition of the membership function of the fuzzy numbers defined to represent the performances, the aggregation operators selected to combine fuzzy performance and crisp weights and finally the defuzzification method are still closely related to the specific application made. Consequently, changing the field of application or the specific problem analyzed the overall model should be set again.

On the other side hybrid approach allows exploiting several strengths. First of all the disclosure of airlines' explicit and tacit needs; it allows to handle also incomplete information characterized by uncertainty and vagueness; it makes possible accounting for multiple perspectives which can be computed and properly aggregate, to detect inconsistent judgments of decision making in weight determination, hence it makes the subjectivity of weights definition relevantly low. No complex calculations are involved, neither cost expensive tools are required for the model implementation. Moreover, this hybrid approach allows to deal with qualitative ranges defined for the indicators insuring to keep track of the variation of aircraft performances avoiding over and/or underestimations. As matter of fact the definition of membership function above these qualitative ranges when the term set of the linguistic variables are defined allows overcoming all these issues and representing aircraft performances in a way that is very close to airlines perceptions avoiding evaluation ambiguity.

To test and to verify the usability of hybrid model in the air transport industry, in the next section an empirical study involving three regional aircraft is illustrated.

Table 3 Hybrid - Strengths and Weaknesses

\begin{tabular}{|c|c|}
\hline STRENGTHS & WEAKNESSES \\
\hline $\begin{array}{l}\text { Possibility to end up with clear and detailed frame of airlines' } \\
\text { needs (explicit and latent) }\end{array}$ & \multirow{4}{*}{$\begin{array}{l}\text { Time spending for data collection and } \\
\text { evaluation }\end{array}$} \\
\hline $\begin{array}{l}\text { Possibility to handle incomplete information, uncertainty and } \\
\text { vagueness }\end{array}$ & \\
\hline Multiple perspective analysis & \\
\hline Transparency & \\
\hline No complex calculation & \multirow{5}{*}{$\begin{array}{l}\text { For performances evaluation residual } \\
\text { dependency of the results from } \\
\begin{aligned} & \text { membership function } \\
& \text { characterization } \\
- & \text { aggregation operators } \\
- & \text { defuzzification method }\end{aligned}\end{array}$} \\
\hline No cost expensive & \\
\hline Reduction of subjectivity in weights definition & \\
\hline Ability to detect inconsistent judgments & \\
\hline $\begin{array}{l}\text { Possibility to overcome the ambiguity of border values of } \\
\text { qualitative ranges }\end{array}$ & \\
\hline
\end{tabular}


Possibility to keep track of gaps between numerical values (no over/under estimations)

\section{Empirical Study}

Choosing new aircraft in order to enlarge an airline's fleet requires a careful cost-benefit analysis. On the other hand to development of a new programme manufacturer needs a massive financial investment. To this aim, air transport operators (airlines and manufacturers) need reliable methodologies to model decision processes. The availability of such models is useful to both airlines and manufacturers. Indeed, given the huge investments connected to the purchase of new aircrafts, whereas airlines pursue the maximization of economic pay back, manufacturers are interested in acquiring information about crucial market requirements in order to constantly realign their offer. In particular, it is assumed that the decision maker intends to enlarge its fleet in order to acquire low-emission aircrafts, coherently to future operating scenarios.

Step 1. The identification of airlines needs is performed considering the case of Airitaly, an Italian company, which was interested to select aircrafts to be purchased within a set of three candidates: Bombardier CRJ1000 (first flight 2008), Sukhoi SSJ100 (first flight 2008), and Embraer ERJ190 (first flight 2004).

Step 2. The formal model setting was focused on the definition of the characteristics of the formal model identifying the evaluation criteria useful to better represent the airline's requirements. In this case the evaluation criteria were derived merging some insights coming from the literature (Boeing, 1985; AEA, 1990) with Airitaly requirements. The overall set of provided criteria was then discussed so that the following final set of criteria was selected: Operative costing, Aircraft price, cruising speed, autonomy, habitability, environmental pollution, noise. For each one of them specific indicators were defined and specifically normalized in a range from 0 to 1 .

Step 3. Collected criteria were organized in homogeneous groups by interviewees involved in the aircraft evaluation process. The output of such gathering is the hierarchy of criteria indicated in figure 2 .

Step 4. Table 4 reports the pair-wise matrix that is the result of the focus group meeting with the Airline decision makers. It represents the priority establishment between the criteria at the first level of the hierarchy according to the Saaty's scale. A similar procedure was followed also for the other elements in the hierarchy.

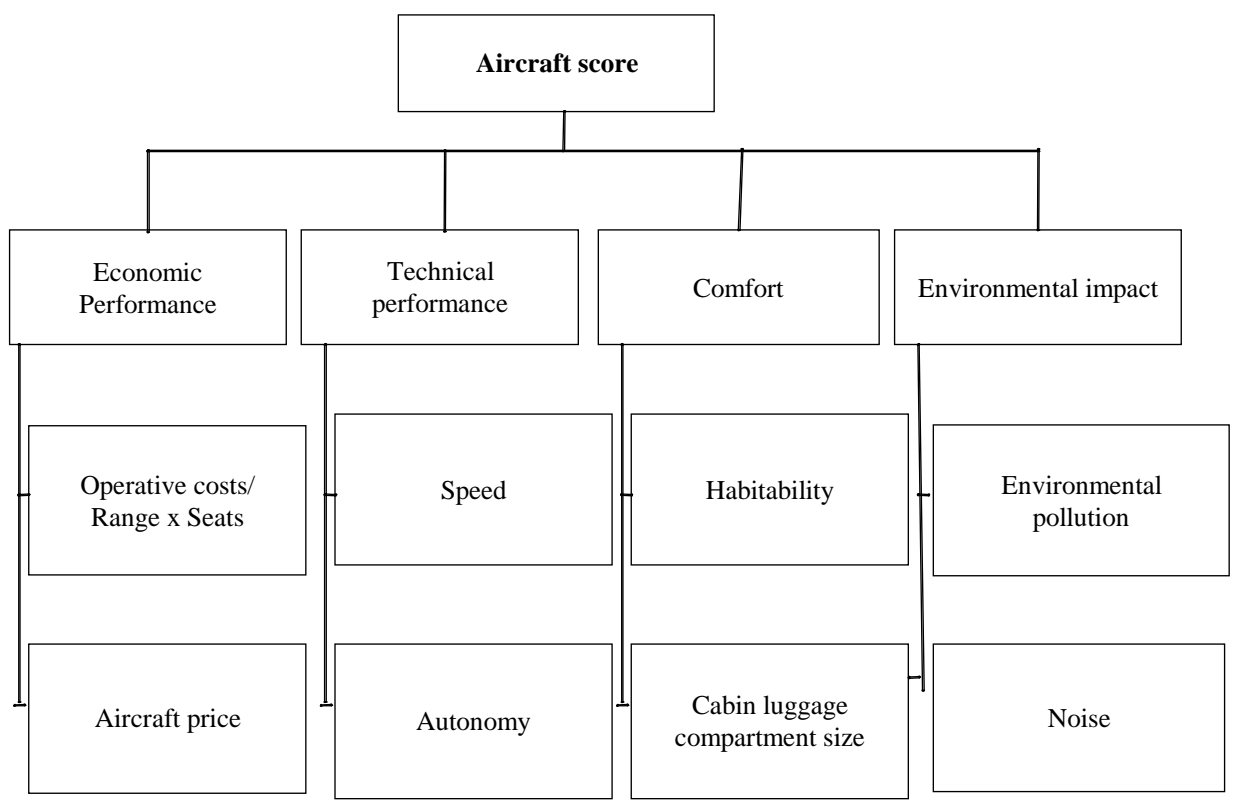

Figure 2. Hierarchy of criteria

Table 4. Pairwise matrix

\begin{tabular}{|c|c|c|c|c|}
\hline $\begin{array}{c}\text { VENDOR } \\
\text { RATING }\end{array}$ & $\begin{array}{c}\text { Economic } \\
\text { performance }\end{array}$ & $\begin{array}{c}\text { Technical } \\
\text { performance }\end{array}$ & Comfort & $\begin{array}{c}\text { Environmental } \\
\text { impact }\end{array}$ \\
\hline $\begin{array}{c}\text { Economic } \\
\text { performance }\end{array}$ & 1.00 & 5.00 & 5.00 & 5.00 \\
\hline $\begin{array}{c}\text { Technical } \\
\text { performance }\end{array}$ & 0.20 & 1.00 & 1.00 & 2.00 \\
\hline Comfort & 0.20 & 1.00 & 1.00 & 2.00 \\
\hline
\end{tabular}




\begin{tabular}{|c|c|c|c|c|}
\hline $\begin{array}{c}\text { Environmental } \\
\text { impact }\end{array}$ & 0.20 & 0.50 & 0.50 & 1.00 \\
\hline
\end{tabular}

Step 5. The principal eigenvalue $\left(\lambda_{\max }\right)$ of the matrix reported in tab.4 was computed to evaluate the consistency index (CI). The last one was, then, matched with random index (RI) to derive the consistency ratio (CR). As reported in table 5 the final consistency ratio was equal to 0,041789 which is less the threshold $(0.1)$ needed to assure consistency.

Step 6. Once the consistency of the matrix has been verified, the final priority vector has been obtained (Table 6$)$. The eigenvector associated to the principal eigenvalue $\left(\lambda_{\max }\right)$, was calculated and normalized to obtain the final priority vector. Its components, as indicated in table 6 , correspond to the weights associated to the variables at the first level of the hierarchy.

Table 5. Principal eigenvalue, consistency index and consistency ratio

Table 6. Priority vector

\begin{tabular}{|c|c|}
\hline \multicolumn{2}{|c|}{ VENDOR RATING } \\
\hline$\lambda_{\max }$ & 4.0606 \\
\hline $\mathrm{CI}$ & 0.020 \\
\hline $\mathrm{RI}$ & 0.890 \\
\hline $\mathrm{CR}$ & 0.023 \\
\hline
\end{tabular}

\begin{tabular}{|c|}
\hline Priority vector \\
\hline 0.62 \\
\hline 0.15 \\
\hline 0.15 \\
\hline 0.09 \\
\hline
\end{tabular}

Following the same procedure, the weights associated to the other variables of the hierarchy were extracted obtaining the final results depicted in figure 3.

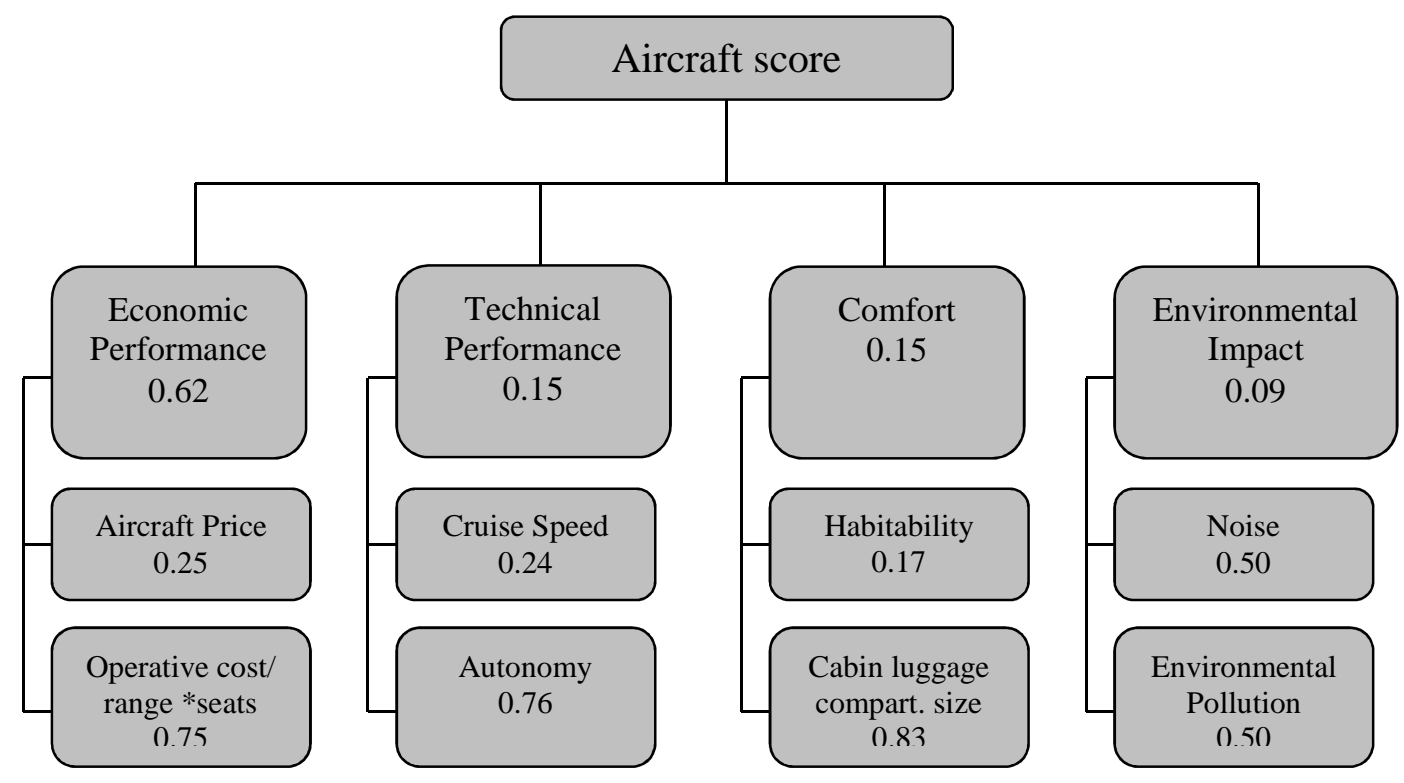

Figure 3. Weights associated to evaluation criteria

Step 7. An example of definition of performances as linguistic variables is reported in the following. The qualitative ranges associated to five levels (very poor, poor, medium, good and very good) defined for the criterion Speed and derived from the judgments collected from four experts interviewed (decision makers, DM) are reported in figure 4. For each level the intersection and the union of the four judgements represent the core and the support of the fuzzy number.

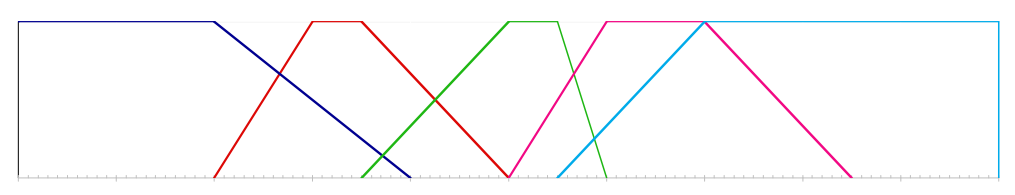

Figure 4. Definition of performance as linguistic variable for the criterion "Speed" 
Step 8 and Step 9. An example of performance fuzzification is reported in reference to the criterion Speed Time. Figure 5 shows that starting from performance evaluation as numerical performances measured trough specific indicators associated to the variables reported in the hierarchy, which in this case is for the CRJ1000 0.40, this performance is then fuzzified comparing the numeric value to the term set of the linguistic variables defined for that criterion.
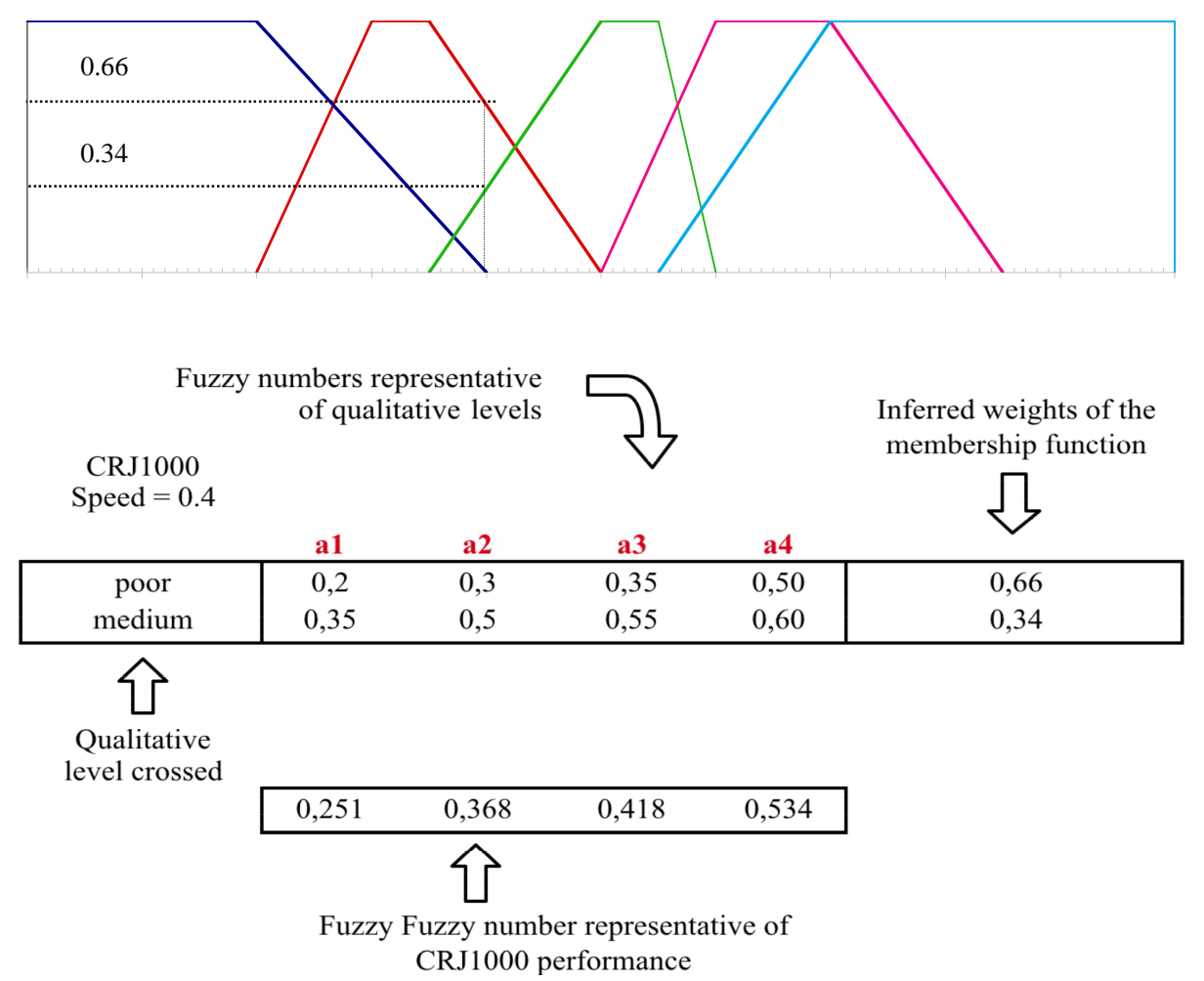

Figure 5. Performance evaluation and fuzzification for the criterion "Speed"

The fuzzy numbers corresponding to crossed membership functions combined with the inferred weights of the members through a fuzzy weighted operator obtaining the final fuzzy number representing CRJ1000 performance for the criterion Speed. This same "fuzzification" method was applied to get fuzzy numbers representative of the performances of the criteria belonging to the last level of the hierarchy and also all the other criteria of the hierarchy which were directly measured to evaluate the score for each aircraft evaluated.

Step 10. Crisp weights and fuzzy performances were aggregated across the hierarchy, according to the aggregation of crisp weights and fuzzy performances to obtain the fuzzy preference index through the weighted fuzzy operator Figure 6, 7 , and 8.

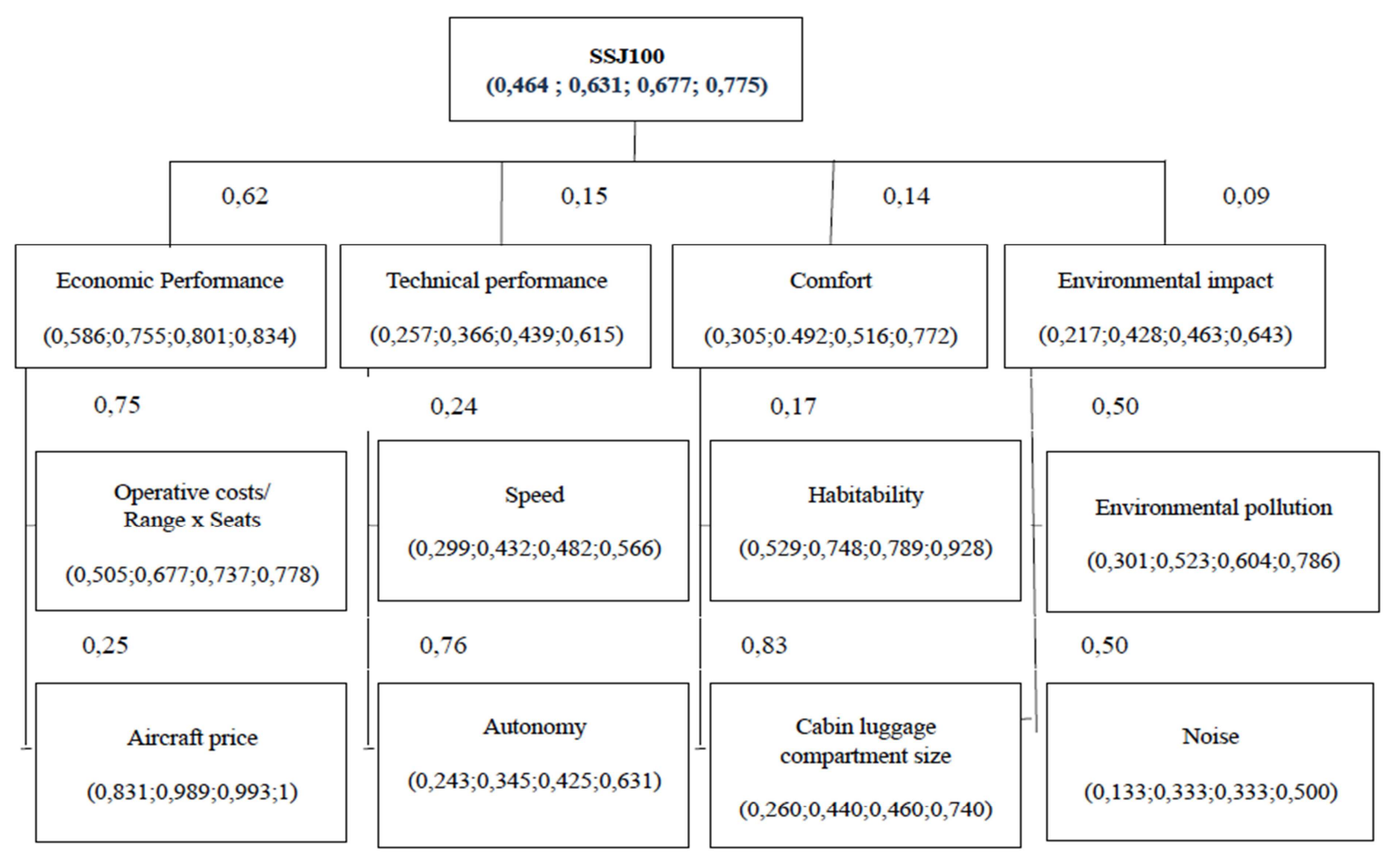




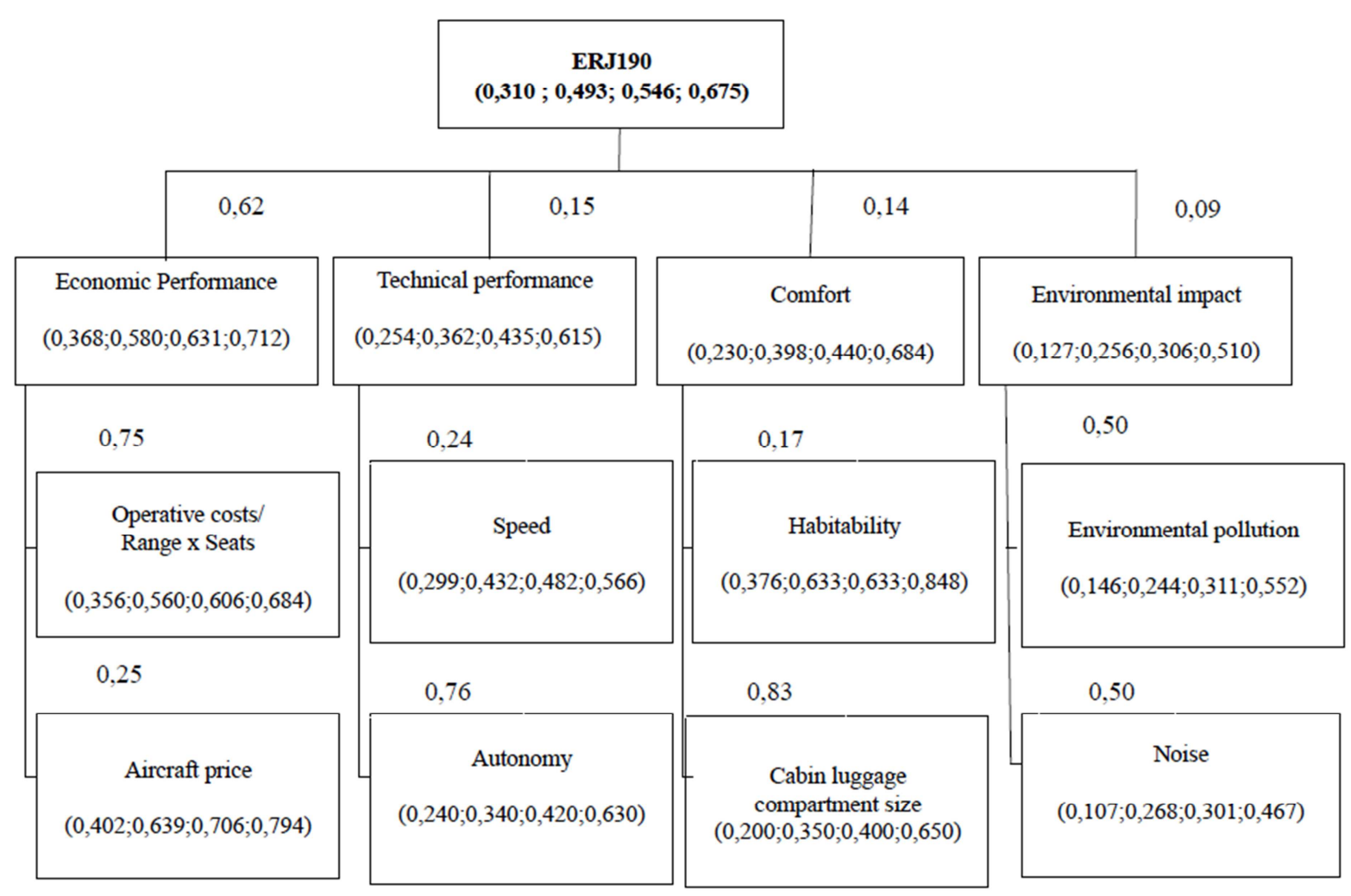

Figure 7. Bombardier CRJ1000

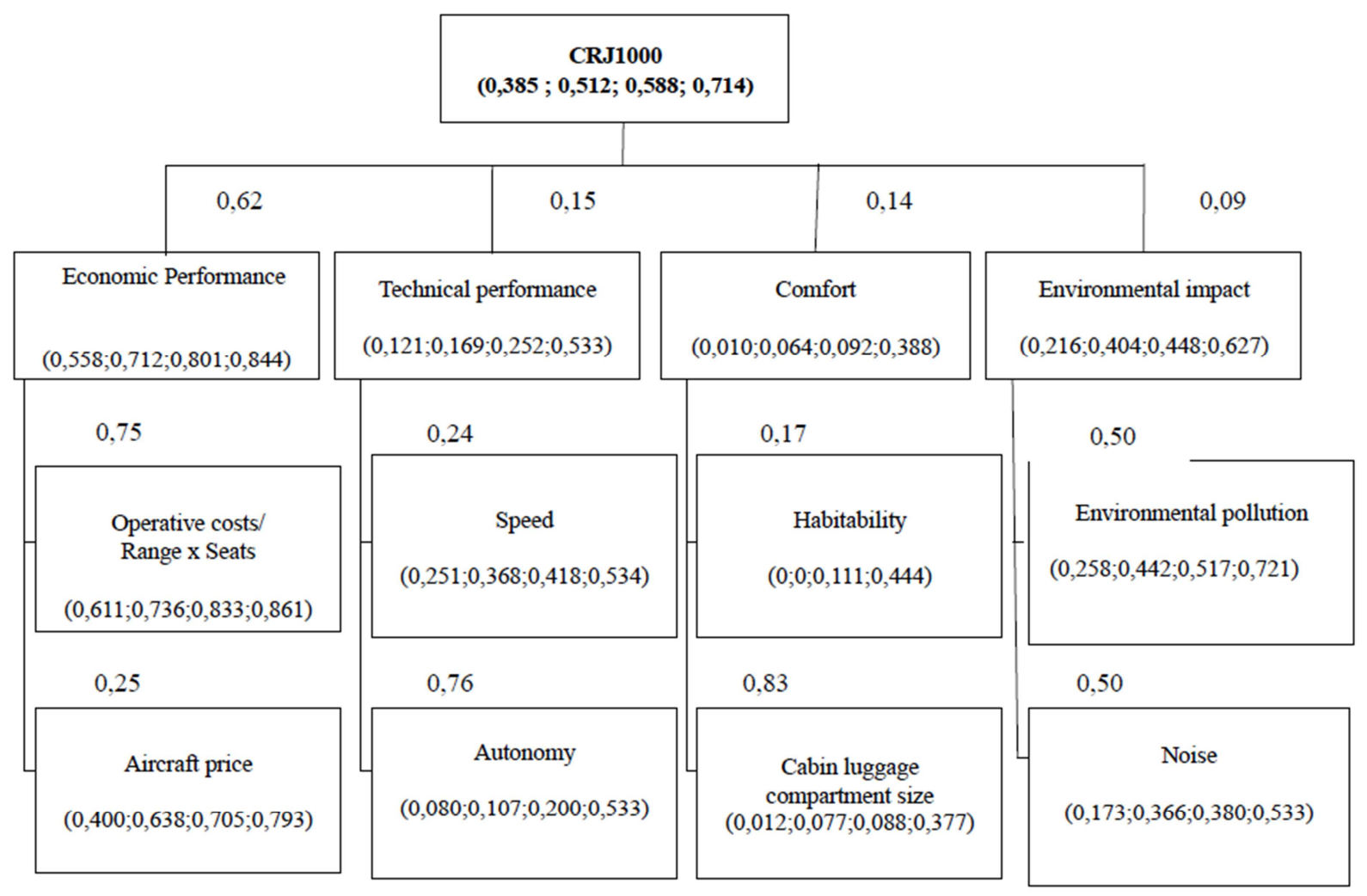

Figure 8. Embraer ERJ190

Step 11. Finally, in adherence to the de-fuzzification and ranking of the fuzzy preference index for each alternative, the fuzzy numbers representative of the vendor ratings were de-fuzzified adopting the middle of maxima (MoM) de-fuzzification method. The results are reported in table 7 .

Table 7. Aircraft final score

\begin{tabular}{|c|c|c|}
\hline Aircraft & Fuzzy rating & Crisp score \\
\hline Sukhoi SSJ1000 & $(0,464 ; 0,631 ; 0,677 ; 0,775)$ & 0.637 \\
\hline Bombardier CRJ1000 & $(0,385 ; 0,512 ; 0,588 ; 0,714)$ & 0.550 \\
\hline Embraer ERJ190 & $(0,310 ; 0,493 ; 0,546 ; 0,675)$ & 0.506 \\
\hline
\end{tabular}


The table shows that the Sukhoi SSJ 1000 has an higher score than the Bombardier CRJ1000 and the Embraer ERJ190. It is important to underline how the method can be used as a strategic tool by both airlines and for manufacturing companies. The practical relevance of the hybrid approach does not consist only in its usefulness as an evaluation system but rather in the opportunity to adopt it as a strategic approach. In fact airlines may use the model to clearly identify their own requirements whereas manufacturers may use the model in the phase of design for better meet the airlines requirements.

For example, the Embraer ERJ190 appears to be the aircraft with the lowest score. However the analysis of the performances (figure 8) provides useful suggestions about how it could be possible to gain positions mainly increasing the value of the sub-criterion "Operative costs", which is definitely lower than others aircraft and whose associated weight is considerably high (0.75).

\section{Conclusions}

In this paper a model for regional aircraft evaluation has been proposed. The model includes four criteria (economic performance, technical performance, comfort, and environmental impact) and eight subcriteria (aircraft price, operative cost, cruise speed, autonomy, habitability, cabin luggage compartment size, noise, and environmental pollution). The model framework is articulated in eleven steps and is based on a hybrid approach that uses both the Analytic Hierarchy Process and the Fuzzy Set Theory. The AHP approach is preserved for criteria weights determination because it ensures to keep track of the differences between importance associated to diverse criteria thanks to pair-wise evaluations between them; the FST approach, instead, is adopted to deal with aircraft performances because it allows representing the vagueness associated to criteria and indicating the "nuances" of airlines perceptions about aircraft performances without losing information. In this way the proposed model overtakes some AHP and FST weaknesses and combines some of their strengths. The model has been tested starting from the requirements of an Italian airline (Airitaly) and considering three potential candidates (Sukhoi SSJ 1000, Bombardier CRJ1000, and Embraer ERJ190) to be selected for enlarge airline's fleet.

The results of the model implementation highlight how practical relevance of the hybrid approach does not consist only in its usefulness as an evaluation system but rather in the opportunity to adopt it as a strategic approach. In particular, airlines may use the model to clearly identify their own requirements whereas manufacturers may use the model for better meet the airlines requirements.

\section{REFERENCES}

AEA (1990). Definition and Input for Range e DOC calculation, Report.

Bernstein, D. (2008). UK's Sustainable Aviation Council, Aviation Week \& Space Technology, May 26.

Boeing (1985). The Boeing Method for Generalized Airplane operating Economics, Boeing, Seattle.

Braathen, N. A. (2005). OECD Experience with Environmental Taxes and Charges, Seminar on Tax Reform Trends Institute for Fiscal Studies, Madrid - 16 May.

Canaday, H. (2005), Cost control over time, Overhaul \& Maintenance, October.

Doganis, R. (2006). The Airliners Business, Edited by Routledge, New York.

ETS (2005). Industry Affaire: Proposal for inclusion of aviation in the EU emission trading scheme, Report of European Trade Scheme.

EU (2007). The European Commission incorporates all airliners into the ETS, January.

FAA (2008). Policy regarding airport rates and charges, FAA official documents.

Ferreri, D. (2003). Marketing and Management, Praeger, Westport, Connecticut USA.

ICAO (2003). Engine Emission Databank, ICAO Report.

Harker, P.T., Vargas, L.G. (1990), Replay to "Remarks on the analytic hierarchy process" by J.S. Dyer, Management Science, 36 (3), 269-273

Hope, J. (2005). Emission from international air-transport and related emission policy, ICAO Report.

McNamara, S. (2008). EU ETS moves to next stage, Regional International Journal, June.

Newson, C., Cairns, S. (2006), Predict and decide: aviation, climate change and UK policy, Report of UK Environmental Change Institute. 
OPEC (2008), Executive summary 2008 - OPEC Document.

Pérez J., Jimeno J.L., Mokotoff E. (2006). Another potential shortcoming of AHP, TOP, v.14 n.1, 99-111

Saaty T.L. (1980). The Analytic Hierarchy Process. McGraw Hill International, New York.

Saaty T.L. (2001). Decision making with Dependence and Feedback- The Analytic Network Process, Second Edition, RWS Publications, Pittsburgh.

Saletic, D., Velasevic, D., Mastorakis, N. (2002). Analysis of Basic Defuzzification Techniques. Proceedings of the 6th WSES International Multiconference on Circuits, Systems, Communications and Computers.

Stonecipher, C. (2008), Economic advantage and Disadvantage, Aviation Maintenance and Repair Industry- Economics, January 20.

Triantaphyllou, E., Mann, S.H. (1995). Using the analytic hierarchy process for decision making in engineering applications: some challenger, International Journal of Industrial Engineering: Applications and Practice, V.2, N.1, 35-44.

Wall R. (2008), Advocating Alternatives, Aviation Week \& Space Technology May 26.

Watanabe, N., (1979). Statistical Methods for Estimating Membership Functions. Japanese Journal of Fuzzy Theory and Systems 5(4).

WRI (2003). Hot climate, cool commerce: a service sector guide to greenhouse gas management, World Resource Institute Report.

Zadeh, L.A., (1965). Fuzzy Sets. Information and Control 8, 338-353. 